\title{
La consulta de predialisis de enfermería: logros y oportunidades de mejora
}

\section{Rosario Álvarez Pérez Sonia Velasco Ballestero}

Unidad de Diálisis Hospital General de Segovia

\section{RESUMEN}

Se realiza un estudio retrospectivo donde se revisan todos los pacientes incidentes en el periodo 2000-2005 en Segovia. El objetivo del mismo es describir el papel que desempeña la Consulta Prediálisis en la elección del tipo de Tratamiento Renal Sustitutivo y en la disponibilidad de un acceso vascular permanente funcionante 0 un catéter peritoneal al inicio de diálisis, así como analizar la situación de nuestros pacientes a su entrada en programa respecto a las vacunaciones.

Se revisan 123 pacientes de los que 99 entraron en HD y 24 en DPCA. Noventa y siete pacientes procedían de la Consulta Prediálisis y fueron seguidos en la misma durante una media de 26 meses. De los 97 pacientes vistos, 76 entraron en HD y 21 en DPCA. En el momento de iniciar HD, un $92 \%$ de los pacientes procedentes de la Consulta Prediálisis disponía de acceso vascular permanente normofuncionante; el $56,5 \%$ de los pacientes iniciaron HD de forma programada. En el momento de iniciar CAPD, los 21 pacientes vistos en la consulta disponían de catéter peritoneal normofuncionante, y todos iniciaron la técnica de forma programada en su domicilio.
En cuanto a la vacunación de hepatitis $B$, de los pacientes no previamente inmunizados, el $70,6 \%$ había completado vacunación y el 20,6\% había recibido al menos dos dosis.

Los resultados muestran que la Consulta Prediálisis favorece la atención de los pacientes con Enfermedad Renal Crónica estadio 5 al permitir la toma con tiempo, por parte del paciente y su familia, de una decisión informada respecto al método de diálisis; la estandarización y protocolización del seguimiento; la implantación con suficiente antelación de un acceso o catéter idóneo; la vacunación de Hepatitis B y en definitiva, la mejora en la eficiencia (costes), la disminución de la morbimortalidad y el incremento en el bienestar de los pacientes.

\author{
Palabras clave: \\ CONSULTA PREDIALSIS \\ HEMODIALISIS \\ DIALISIS PERITONEAL \\ ACCESO VASCULAR \\ CATETER \\ VACUNACIÓN \\ ELECCION TRATAMIENTO
}

\section{Correspondencia: \\ Rosario Álvarez Pérez \\ Unidad de Hemodiálisis \\ Hospital General de Segovia \\ Carretera de Ávila, s/n \\ 40002 Segovia \\ malvarez@hgse.sacyl.es}




\section{Nursing predialysis clinic: achievements and opportunities for improvement}

\begin{abstract}
Retrospective study reviewing the characteristics and evolution of all incident patients in Segovia, during the period 2000-2005. The main objective was to describe the role of the Predialysis Clinic (PDC) in the selection of the kind of dialysis (hemo or peritoneal), the availability of a functioning access (either a vascular access or a peritoneal catheter) at the beginning of treatment, and in hepatitis $B$ vaccination.
\end{abstract}

We reviewed 123 patients of who 99 started haemodialysis (HD) and 24 Chronic Ambulatory Peritoneal Dialysis (CAPD). 97 patients were seen in the PDC and were followed up for a mean period of 26 months (range 1-126). Of them, 76 started HD and 21 CAPD. At the time of starting hemodialysis, $92 \%$ of the patients seen in the PDC had a functioning vascular access and $56,5 \%$ of them started as outpatients (not needing hospitalization). At the time of starting CAPD, all 21 patients had a functioning peritoneal catheter and all of them started as outpatients.

Twenty two patients had hepatitis B antibodies. $70,6 \%$ of the patients no previously inmmunized had received four doses and $20,6 \%$ had received at least two doses of hepatitis $B$ vaccination, prior to starting on dialysis.

This results shows that PDC improves the attention to patients with Stage 5 Chronic Renal Disease since: it permits to take an informed decision, by the patient and its family, of the preferred kind of dialysis; it facilitates the standardization of follow-up; it permits the timely construction of an adequate vascular access or peritoneal catheter, as well as the complete Hepatitis B vaccination; and, in summary, improves efficiency, diminish morbimortality and preserves patients wellbeing.

\author{
key words \\ PREDIALYSIS CLINIC \\ HAEMODIALYSIS \\ PERITONEAL DIALYSIS \\ VASCULAR ACCESS \\ CATHETER \\ VACCINATION \\ TREATMENT ELECTION
}

\section{Introducción}

El tratamiento sustitutivo de la función renal en los pacientes con enfermedad renal crónica estadio 5 (ERC5) ha evolucionado de manera importante en las últimas décadas gracias a los grandes avances científicos y tecnológicos que han afectado tanto a la técnica como al tratamiento médico.

La universalización en algunos países, entre ellos España, de la asistencia sanitaria y el aumento de la disponibilidad de recursos han permitido ampliar el número de pacientes susceptibles de ser tratados con diálisis, de modo que en la actualidad no existen barreras, al menos desde el punto de vista de las autoridades sanitarias, para aplicar la diálisis a cualquier paciente que lo precise ${ }^{1}$. Esto explica que la incidencia haya ido aumentando a expensas de la entrada en programa de pacientes cada vez más ancianos y con mayor comorbilidad.

Distintos autores han alertado sobre la repercusión del retraso en la llegada a las consultas de Nefrología de los pacientes con ERC. Una de las consecuencias es que un elevado porcentaje de pacientes, al carecer de acceso vascular (AV) definitivo, tiene que iniciar hemodiálisis (HD) a través de un catéter central transitorio (CCT), lo que tiene una importante repercusión en los costes, la morbi-mortalidad y el bienestar del paciente ${ }^{2}$. Un análisis de la situación de los AV en Segovia, en el periodo 1991-2000, mostraba que los pacientes vistos en Consulta de Prediálisis (CPD) que eran educados acerca de su situación y que participaban en la toma de decisiones disponían con mayor frecuencia de $\mathrm{AV}$ al inicio de $\mathrm{HD}^{3}$. 
Desde la apertura de la Unidad en enero de 1986, todos los pacientes con aclaramiento de creatinina $<30 \mathrm{ml} / \mathrm{min}$ se revisan en la CPD. Se trata de una consulta mixta (médico y enfermera por separado) en la que se realiza consejo dietético y control del estado nutricional, se ayuda a los pacientes a tomar una decisión informada sobre la necesidad de tratamiento renal sustitutivo (TRS), se toman las medidas necesarias para que el paciente disponga de un AV o catéter peritoneal adecuado al inicio del programa, se realiza vacunación de hepatitis $B$ y control de respuesta, y se actúa en otras áreas como por ejemplo la enseñanza para el tratamiento con eritropoyetina subcutánea (EPO) y el manejo de la hipertensión arterial (HTA) y/ o la diabetes ${ }^{4-5}$.

\section{Objetivo}

El objetivo de este estudio es describir el papel que desempeña la CPD en la elección del tipo de TRS y en la disponibilidad de un AV permanente funcionante 0 un catéter peritoneal al inicio de diálisis, así como analizar la situación de nuestros pacientes a su entrada en programa respecto a las vacunaciones.

\section{Material y métodos}

Se realizó un estudio retrospectivo donde se revisaron todos los pacientes incidentes en el periodo 2000-2005 en Segovia. En cada caso se analizaron las siguientes variables: fecha de entrada en consulta y aclaramiento de cretinina (Ccr); fecha de entrada en programa; quién decide el tipo de tratamiento; edad al inicio de TRS; causa de la ERC; existencia de hipertensión arterial (HTA) y/ o antecedentes cardiovasculares documentados en la historia clínica; Índice de Comorbilidad (IC) de Charlson; procedencia: consulta de prediálisis o urgentes; fecha de realización, tipo, localización y estado del AV o catéter peritoneal a su entrada en programa; número de dosis de vacuna de hepatitis $B$ recibidas, tratamiento con EPO y/ o hierro intravenoso y consejo dietético.

\section{Resultados}

Se revisaron 123 pacientes (72 hombres y 51 mujeres) de los que 99 entraron en HD y 24 en DPCA. En las tablas 1 y 2 se presenta la evolución anual de las características clínicas de los pacientes incidentes según el tipo de diálisis. En la tabla 3 se comparan las características clínicas de ambas poblaciones: los pacientes incluidos en HD tenían más edad y mayor comorbilidad.

\begin{tabular}{|l|l|l|l|l|l|}
\hline Año & Edad & DM (\%) & ACV (\%) & IC & CPD (\%) \\
\hline $91-00$ & 64 & 20,0 & - & - & 85,9 \\
\hline 2000 & 70 & 37,5 & 50,0 & 7,5 & 87,5 \\
\hline 2001 & 73 & 33,3 & 61,1 & 8 & 78,8 \\
\hline 2002 & 71 & 35,0 & 65,0 & 8 & 70,0 \\
\hline 2003 & 74 & 61,1 & 39,0 & 8 & 84,3 \\
\hline 2004 & 72 & 20,0 & 66,7 & 8 & 66,7 \\
\hline 2005 & 71 & 33,3 & 50,0 & 7,5 & 66,7 \\
\hline
\end{tabular}

Tabla 1. Pacientes incidentes en Hemodiálisis. Medianas y porcentajes

ACV: Antecedentes documentados de insuficiencia cardiaca, cardiopatía isquémica, enfermedad vascular cerebral y/o enfermedad vascular periférica

DM: Diabetes méllitus

IC: Índice de Comorbilidad de Charlson modificado CPD: Consulta Prediálisis

\begin{tabular}{|l|l|l|l|l|l|}
\hline Año & Edad & DM (\%) & ACV (\%) & IC & CPD (\%) \\
\hline 2000 & 69,0 & 33,3 & 66,7 & 7 & 100 \\
\hline 2001 & 49,5 & 25 & 25,0 & 4,5 & 100 \\
\hline 2002 & 49,0 & 50 & 25,0 & 4,0 & 100 \\
\hline 2003 & 64,0 & 0 & 33,3 & 6 & 100 \\
\hline 2004 & 47,0 & 50 & 16,7 & 5,5 & 100 \\
\hline 2005 & 58,5 & 50 & 25,0 & 6,5 & 100 \\
\hline
\end{tabular}

Tabla 2. Pacientes Incidentes en DPCA. Medianas y porcentajes

ACV: Antecedentes documentados de insuficiencia cardiaca, cardiopatía isquémica, enfermedad vascular cerebral y/o enfermedad vascular periférica

DM: Diabetes méllitus

IC: Índice de Comorbilidad de Charlson modificado

CPD: Consulta Prediálisis

\begin{tabular}{l|l|l|l|l|l|l|}
\hline Tipo & Número & Edad & HTA & ACV & DM & IC \\
\hline HD & 99 & $69,3 \pm 11,9$ & $67,7 \%$ & $55,6 \%$ & $59,7 \%$ & $8,1 \pm 2,3$ \\
DPCA & 24 & $54 \pm 13,4$ & $83,3 \%$ & $29,2 \%$ & $37,5 \%$ & $6,0 \pm 2,9$ \\
p & & $<0,001$ & n.s. & $<0,05$ & n.s. & $<0,001$ \\
\hline
\end{tabular}

Tabla 3. Comparación según tipo de diálisis. Medias \pm DS y porcentajes

HTA: Hipertensión arterial

ACV: Antecedentes documentados de insuficiencia cardiaca, cardiopatía isquémica, enfermedad vascular cerebral y/o enfermedad vascular periférica

DM: Diabetes méllitus

IC: Índice de Comorbilidad de Charlson modificado 
Noventa y siete pacientes procedían de la CPD $(78,9 \%)$ y $26(21,1 \%)$ fueron considerados urgentes (23 HD, 3 DP). Los pacientes de la CPD tenían en el momento de su entrada en la consulta un $\mathrm{Ccr}$ medio de $22 \mathrm{ml} / \mathrm{min}$ (rango 9 a $46 \mathrm{ml} / \mathrm{min}$.) y fueron seguidos en la misma durante una media de 26,0 meses (rango 1-126 meses).

A todos los pacientes revisados en la CPD se les explicó qué es la ERC y cuáles eran los métodos de TRS en su caso, así como se les apoyó en la toma de decisión; se realizó consejo (restricción proteica, de sal y/o de potasio según necesidades) y seguimiento nutricional, y en el caso de los pacientes diabéticos, obesos e hipertensos se adaptó la enseñanza a sus necesidades particulares.

En todos los pacientes se revisaba en cada consulta la medicación prescrita, se les enseñaba para qué y cómo tomarla y se les suministraba una hoja de tratamiento actualizada. Treinta y cinco pacientes recibían tratamiento con hierro intravenoso: a todos ellos se les realizó el test de Venofer ${ }^{\oplus}$; en aquellos que tenían problemas de acceso venoso se les administró el Fe en la Unidad y al resto en sus Centros de Salud según protocolo ${ }^{6}$. Cincuenta y cuatro pacientes recibían EPO; a todos ellos se enseño la técnica de administración.

De los 97 pacientes vistos en CPD, 76 (78,3\%) entraron en HD y $21(21,6 \%)$ en DPCA. El porcentaje de pacientes incidentes incluidos en DPCA osciló entre el $14 \%$ y el $18 \%$ en el periodo $2000-2003$; en 2004 se actualizaron los protocolos de DPCA y se revisó la metodología de información a los pacientes, aumentando a partir de entonces el porcentaje de los que se decidieron por DPCA $(28,6 \%$ en 2004 y $25,0 \%$ en 2005). La decisión sobre el tipo de TRS fue tomada por el médico en 17 ocasiones, por el paciente en 69 y en 11 conjuntamente entre médico y paciente.

El $56,5 \%$ de los pacientes vistos en la CPD iniciaron HD de forma programada (no precisaron ingreso inicial). En el momento de iniciar HD, los 76 pacientes de la CPD disponían de AV permanente (4I FAV radio-cefálicas; 15 húmero-cefálicas; 7 húmero-basílicas; 12 prótesis de Goretex y un catéter permanente).
De las 75 FAV/Prótesis, 69 eran normofuncionantes al inicio de diálisis; en cuatro casos estaban inmaduras y en dos trombosadas lo que condicionó el uso de catéteres temporales, en su mayoría de doble luz por vía femoral. En la tabla 4 se muestra el tipo de AV inicial en el conjunto de los pacientes incidentes (CPD y urgentes). En la tabla 5 se muestra el porcentaje de los AV (FAV y prótesis) normofuncionantes al inicio del TRS.

\begin{tabular}{lllll} 
& FAV & Prótesis & CP & CT \\
\hline 2000 & 14 & 0 & 0 & 2 \\
2001 & 13 & 2 & 1 & 2 \\
\hline 2002 & 10 & 3 & 0 & 7 \\
2003 & 11 & 3 & 0 & 4 \\
2004 & 9 & 1 & 0 & 5 \\
2005 & 9 & 0 & 0 & 3
\end{tabular}

Tabla 4. Pacientes incidentes según acceso vascular

CP: Catéter Permanente

CT: Catéter Temporal

\begin{tabular}{|c|c|c|}
\hline AÑO & $\%$ & \\
\hline $91-00$ & 81,8 & \\
\hline 2000 & 87,5 & \\
\hline 2001 & 83,3 & \\
\hline 2002 & 65,0 & \\
\hline 2003 & 77,8 & \\
\hline 2004 & 66,7 & $\begin{array}{l}\text { labla 5. \% de pacientes con AV } \\
\text { (FAV o prótesis) normofuncio- }\end{array}$ \\
\hline 2005 & 75,0 & nante al inicio de HD. \\
\hline
\end{tabular}

En el momento de iniciar CAPD, los 21 pacientes vistos en la CPD disponían de catéter peritoneal normofuncionante, y todos iniciaron la técnica de forma programada en su domicilio.

De los 97 pacientes vistos en la CPD, 22 pacientes $(22,7 \%)$ estaban inmunizados. De los 75 pacientes restantes, en el momento de iniciar la diálisis 53 (70,6\%) habían recibido las cuatro dosis de $40 \mathrm{mcg}$ de Engerix B, (vacuna hepatitis) y 15 pacientes $(20,6 \%)$ habían recibido entre dos y tres dosis; sólo en siete casos $(9,3 \%)$ no se había iniciado la vacunación. 


\section{Discusión y conclusiones}

Este estudio muestra que un alto porcentaje de pacientes que inician TRS en Segovia han sido vistos durante meses en la CPD. Consideramos que esto ha favorecids la entrada de los pac entes en HD con un AV defin tivo norm ofuncionante como yć se mostró en un estıdio anter ior ${ }^{2}$. Estos resultados si? comparan muy favo ablemente con lo publicado, sieıdo lo habitual que $! n$ el $45-55 \%$ de los pacientes $h$ aya que recurrir a a implan ación de un catéter temporal al inicio de tratamier $t^{5}$.

Si comparamos nuestros resultados en el periodo 1991-2000 con los actuales (tablas 4 y 5) cabe destacar que el porcentaje de pacientes vistos en CPD ha ido disminuyendo mientras que ha aumentado el de aquellos que entran de forma urgente, lo que podría ser debi xo, en parte, a la tardanza en remitir al paciente a $\Lambda$ efrología, tanto desde Atención Primaria como de ide otros Servicios Hospitalarios. Esto explica que I número de AV (FAV y prótesis) normofuncionantes al inicio de HD haya ido decreciendo, mientras aumentaba el de catéteres temporales. Por otra par :e el incremento en la edad y la comorbilidad y sobre todo el alto porcentaje de pacientes diabéticos cın patología vascular asociada explica las crecientes dificultades para conseguir y mantener un AV permanente adecuado.

En el periodo analizado el $92 \%$ de los AV eran normofuncionantes al inicio de HD. La situación de inmadurez de cuatro AV fue debida en tres ocasiones al deterioro rápido de la función renal de los pacientes y en la cuarta a que el paciente llevaba sólo un mes siendo revisado en CPD. De los dos AV trombosados se tenía constancia pero, dadas las características de los pacientes, se decidió posponer la intervención hasta su entrada en programa.

Un factor para conseguir que los pacientes que entren en HD dispongan de un AV funcionante es que éste se realice con la suficiente antelación. En las guías DOQI7 se recomienda que la cirugía se programe cuando el $\mathrm{Ccr}$ es inferior a $25 \mathrm{ml} /$ min permitiendo un periodo de maduración de 3 ó 4 meses. En nuestro estudio la media de antelación para la reali- zación del AV fue de ocho meses, resultado por encima de nuestros datos de estudios anteriores, donde era de cinco meses.

En lo referente al área de Diálisis Peritoneal, como es habitual, los pacientes tenían una media de edad y un IC menor que los pacientes de HD. Todos los que habían sido vistos en la CPD disponían de catéter peritoneal normofuncionante. Como se ha descrito anteriormente, en 2004 se actualizaron los protocolos de DPCA y se revisó la metodología de información a los pacientes, aumentando a partir de entonces el porcentaje de los que se decidieron por esta técnica ( $28,6 \%$ en 2004 y $25,0 \%$ en 2005 ) lo que nos aproxima a las CCAA con las mayores incidencias en DPCA.

Otro dato analizado fue quien tomaba la decisión sobre el tipo de TRS. En el $71,1 \%$ de las ocasiones ésta fue tomada por el paciente. Todos los pacientes procedentes de la CPD que eligieron DPCA lo hicieron por decisión propia. El 69,5\% de los pacientes que optaron por HD lo decidieron de forma autónoma y el resto fue por decisión conjunta o facultativa.

Llama la atención que casi la mitad de los pacientes vistos en la CPD iniciaron HD precisando ingreso inicial mientras que en DPCA todos iniciaron la técnica de forma programada en su domicilio. Probablemente esto se justifica por la mayor edad y comorbilidad de los pacientes que inician HD y quizás por una falta de información adecuada y suficiente al paciente para que inicie el tratamiento en las mejores condiciones posibles y no cuando su situación obliga al ingreso previo.

En cuanto a la vacunación de hepatitis $B$, de los pacientes no previamente inmunizados el 70,6\% había completado vacunación y el $20,6 \%$ había recibido al menos dos dosis; sólo en el $9,3 \%$ de los casos no se había iniciado la vacunación. En algunos casos la falta de tiempo para completar vacunación y en otros el olvido de alguna de las dosis por parte del paciente 0 de enfermería dadas las características de la vacunación (cuatro dosis separadas en el tiempo) ha dado lugar a este hecho. Pensamos que estos valores deben ser mejorados dado que estos pacientes se consideran grupo de riesgo ante la hepatitis B y son prioritarios para recibir la vacuna. 
Por tanto, y a pesar de los buenos resultados obtenidos, consideramos que debemos hacer un especial énfasis en conseguir las siguientes mejoras:

1. Aumentar el porcentaje de pacientes vistos en CPD, mejorando la comunicación con Atención Primaria y otros Servicios Hospitalarios, para que remitan al paciente con tiempo para poder realizar una preparación adecuada.

2. Aumentar el porcentaje de pacientes vacunados contra la hepatitis $B$ de forma completa.

3. Mejorar la información a los pacientes (en lo referente a sintomatología, necesidad de inicio de tratamiento sustitutivo, etc.) para evitar los ingresos urgentes previos a la entrada en programa.

4. Seguir aumentando el porcentaje de pacientes que deciden tratamiento de DPCA

En resumen consideramos que la CPD favorece la atención de los pacientes con ERC5 al permitir la toma con tiempo, por parte del paciente y su familia, de una decisión informada respecto al método de diálisis; la estandarización y protocolización del seguimiento; la implantación con suficiente antelación de un acceso ( $\mathrm{AV}$ o catéter) idóneo; la vacunación de Hepatitis $B$ y en definitiva, la mejora en la eficiencia (costes), la disminución de la morbimortalidad y el incremento en el bienestar de los pacientes.

\section{Agradecimiento}

Este trabajo ha sido posible, gracias a nuestras compañeras que durante tantos años han dejado constancia escrita de su trabajo.

\section{Bibliografia}

1. Unidad de Nefrología y Comité de Ética Asistencial: Orientaciones para la entrada/ retirada del tratamiento renal sustitutivo. Documento interno del Consorci Hospitalari del Parc Taulí. Sabadell. 1997.

2. Stehman $C$ et al. Determinants of type and timing of initial permanent haemodialysis vascular access. Kidney int. 2000; 57:639-645.

3. Álvarez-Ude F, Álvarez R, Velasco S. Disponibilidad de acceso vascular permanente al inicio de hemodiálisis: papel de la consulta de prediálisis. Nefrología. 2001; 21(6):588-591

4. Velasco S, Álvarez: R. Plan de cuidados estandarizado de las primeras hemodiálisis dirigido a pacientes vistos en consulta de enfermería. Rev Soc Esp Enferm Nefrol. 1999; III Trimestre (7): 51-53.

5. Rodríguez Hernández JA, López Pedret J, Piera L. El acceso vascular en España: análisis de su distribución, morbilidad y sistemas de monitorización. Nefrología. 2001; 21(1): 45-51.

6. Yáñez F, García A. Administración de hierro intravenoso en pacientes en prediálisis. En: Libro de comunicaciones del XXX Congreso de la Sociedad española de Enfermería Nefrológica; Málaga octubre 2005. Barcelona: HOSPAL; 2005.

7. National Kidney Foundation: Dialysis Outcomes Quality Initiative (DOQI). Am J Kidney Dis. 1997; 30(supl 3):S1-S100.

8. Martín Espejo JL, Guerrero Riscos MA. Qué demanda el paciente de prediálisis de la Consulta de Enfermería. Nuestra experiencia. Rev Soc Esp Enferm Nefrol. 2002; II Trimestre (18):17-21. 